\title{
Circulating microRNA-21 as Stable Blood-Based Markers for Patients With ISR After PCI
}

\author{
Xiong Zhang, ${ }^{1}$ Xiao-Ping Xiao, ${ }^{2}$ Xu- Ai Ren, ${ }^{3}$ Wei Xue ${ }^{4}$ \\ ${ }^{1}$ Department of Laboratory, the First People's Hospital of Xian Yang City, Shaanxi Province, China, 712000; ${ }^{2}$ Department of Laboratory, \\ the Eighth Hospital of Xi An City, Shaanxi Province, China, 710061; ${ }^{3}$ Department of Cardiology, the First People's Hospital of Xian \\ Yang City, Shaanxi Province, China, 712000; ${ }^{4}$ Department of Blood Transfusion, the First People's Hospital of Xian Yang City, Shaanxi \\ Province, China, 712000
}

\section{ABSTRACT}

It recently has been reported that the in-stent restenosis (ISR) of expanded area after percutaneous coronary intervention (PCI) within six months can become a serious postoperative complication. A real-time quantitative PCR was used to analyze the expression of serum miR-21 in 33 ISR and 37 non-ISR patients after PCI. Expression of miR-21 was significantly higher in the ISR group compared with that in the NISR group, and a similar trend also occurred in factor- (TNF- $\alpha$ ) level, Interleukin -6 (IL-6) level, and plaque area (PLA). However, a contrary trend occurred in the external elastic membrane area (EEM) and minimal lumen area (MLA). This study suggests that the increased expression of serum miR-21 is related to ISR after PCI, and miR-21 can be a new predictor of ISR.

\section{INTRODUCTION}

Coronary stenting is one of the important methods to treat coronary artery stenosis caused by ischemic heart disease, including angina or acute coronary events [Li-Sha 2015; Ren 2008]. In recent years, PCI has been widely used in the treatment of coronary heart disease in China. However, ISR after PCI is still an unsolved clinical problem.

It has been reported that the clinical incidence of ISR after bare metal stent implantation is about $20-35 \%$. The use of drug-eluting stents further reduced the incidence of ISR to $5-10 \%$ [Kim 2011]. Known risk factors for ISR include diabetes mellitus, renal insufficiency, small vessel diameter (3 mm), treatment of complex lesions (B2/C) [Ryan 1988], chronic total occlusion, venous bypasses, ostial or bifurcated lesions, stent undersizing or underexpansion, and necessary implantation of more stents [Kim 2011].

MicroRNAs (miRNAs, miRs) are small (22-nucleotide) noncoding RNAs. They regulate gene expression at the posttranscriptional level by binding to the target mRNA, leading

Received August 4, 2020; accepted August 25, 2020.

Correspondence: Wei Xue, Department of Blood Transfusion, the First People's Hospital of Xian Yang City, Shaanxi Province, China, 712000; +8613609109862 (e-mail: $3383418063 @ q q . c o m)$. either to mRNA degradation or to translational repression [Small 2011]. MiRNAs have emerged as important regulators of several physiological and pathophysiological processes in cardiovascular disease [Fichtlscherer 2011].

Some reports have shown that miRNAs may present diversity of expression in ischemic heart disease and be involved in the pathophysiological process [Qiang 2013]. So far, more than 1,000 miRNAs have been discovered [Bentwich 2005]. In patients with stable coronary heart disease, the circulating levels of miR-126, miR-17 and miR-92a were significantly lower than those in healthy subjects [Fichtlscherer 2010]. Recent studies have shown that miRNA-21 level is related to the degree of coronary artery stenosis. MiRNA-21 level
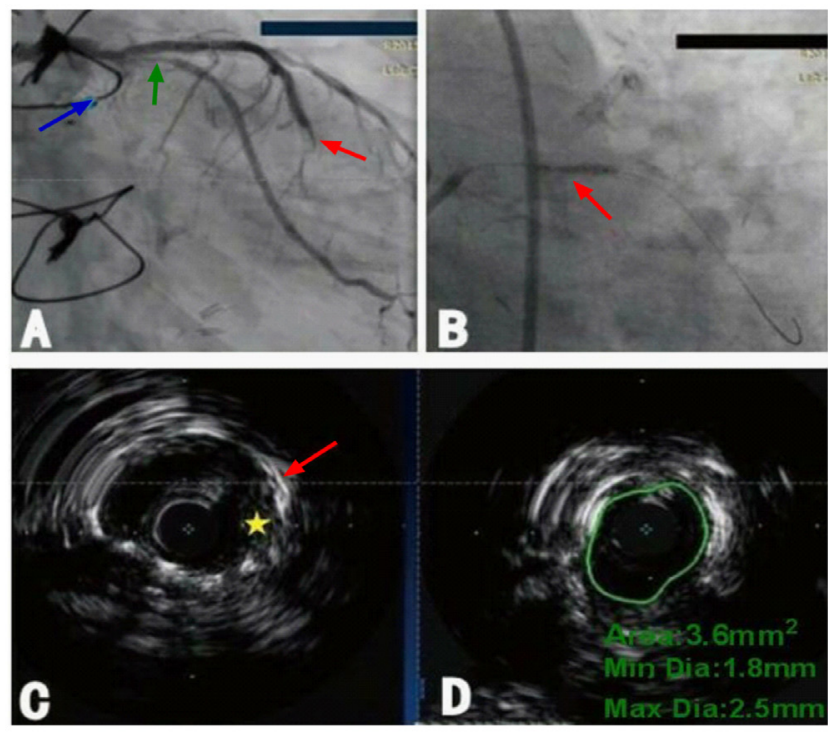

Figure 1. A, Coronary angiogram show severe ISR at proximal Left circumflex (blue arrow) with no flow, proximal Ramus intermedius (green arrow) with slow flow, and mid Left anterior descending artery (red arrow); B, Dilation of the Ramus intermedius coronary artery with 2.5 $\mathrm{mm} \times 22 \mathrm{~mm}$ Non-compliant balloon with 22 atm inflation pressures was done; C, IVUS imaging of the underexpanded stent in the proximal left circumflex lesion show stent struts (red arrow) with evidence of neointimal hyperplasia (yellow star); D, IVUS imaging of the underexpanded stent in the proximal Left circumflex lesion show small stent cross-sectional area of only $3.6 \mathrm{~mm}^{2}$. 

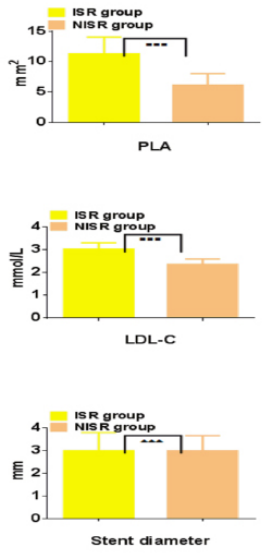

Figure 2. Comparison of Clinical characteristics and the parameters of IVUS for the patients after PCI between the ISR group and the NISR group.

, $P<.01 ; \mathbf{\Delta} \mathbf{\Delta} \mathbf{\Delta}, P>$.05. TG: triglyceride; LDL-C: low density lipoprotein cholesterol; TC: high density lipoprotein cholesterol; TNF- $\alpha$ : Tumour necrosis factor- $\alpha$; IL-6: interleukin-6; TC:Total Chol; IVUS: Intravascular ultrasound; EEM: external elastic membrane; PLA: plaque area; MLA: minimal lumen area

in the acute myocardial infarction group was significantly higher than those in the control group [Yang 2015]. There is evidence that silencing miRNA-21 expression reverses the protective effect of trimetazidine on myocardial ischemiareperfusion injury [Ma 2016].

The purpose of this study is to explore whether miR-21 can be used as a biological index to determine coronary artery stenosis after PCI.

\section{MATERIALS AND METHODS}

Study population: We included 70 patients with acute coronary syndrome who underwent PCI in our hospital cardiology department between March 2018 and March 2020. Within six months after PCI, 33 cases were diagnosed as ISR and 37 cases as non-ISR. The diagnosis of ISR was confirmed by coronary angiography. ISR was defined as recurrent diameter stenosis at the stent segment more than $50 \%$ of the determined vessel diameter [Bentwich 2005; Mitchell 2008].

Intravascular ultrasound (IVUS) was used to evaluate the changes of pathological vessels. Acute coronary syndrome was confirmed in the patient's medical records, including all available clinical records (electrocardiograms, noninvasive imaging studies, coronary angiography, and cardiothoracic surgical reports). The excluded diseases included severe hepatorenal insufficiency, infection, tumor and immune deficiency. After PCI, patients took aspirin $(300 \mathrm{mg} / \mathrm{d})$ and Plavix (75 mg/d) for one month. The study was approved by the ethics committee of our hospital and informed consent was obtained from all patients.

Methods: The expression of miR-21 in serum of patients after PCI was detected by real-time quantitative PCR. According to the manufacturer's instructions (ELISA kit; R \& D system, Minneapolis, Minnesota), the serum concentrations of IL- 6 and TNF- $\alpha$ were detected by ELISA. IVUS was used to evaluate the effect of PCI, ROC curve was drawn,
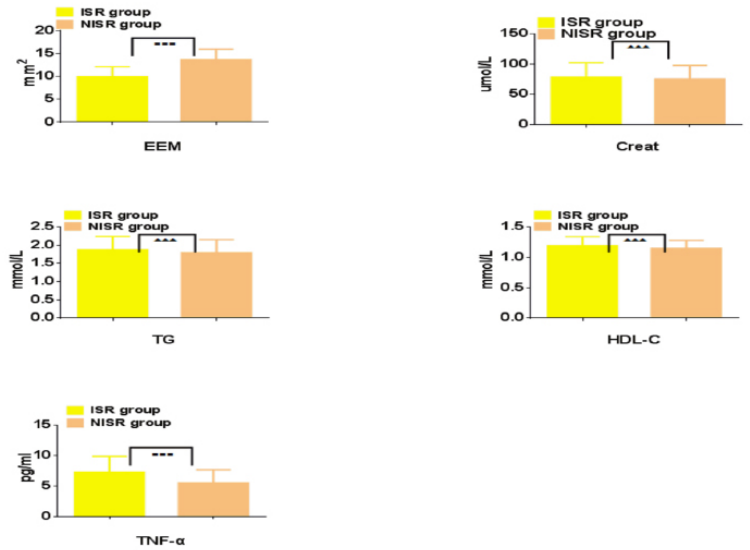

and AUC was calculated for assessing the predictive value of miR-21 to ISR.

Intravascular ultrasound: IVUS is considered a basic coronary imaging method to evaluate ISR and can easily evaluate the characteristics of stent and operation [Alraies 2017]. All subjects received IVUS examination at the sixth month after PCI. IVUS examination was performed on the vascular segment implanted with stent and used a catheter diameter of $2.5 \mathrm{~F}$ as well as the probe frequency of $40 \mathrm{MH}$. The external elastic membrane (EEM), minimal lumen area (MLA), and plaque area (PLA) of stent placement location were measured. IVUS analyses were performed through the agreement of two independent cardiologists (Dr. Yang and Dr. He). The IVUS images are shown in Figure 1.

Specimen collection: Blood samples of patients were collected on the day of IVUS and centrifuged at $3000 \mathrm{rpm}$ at $4^{\circ} \mathrm{C}$ for 15 minutes. The supernatant was transferred to sterile Eppendorf tubes and stored at $-80^{\circ} \mathrm{C}$.

Quantitative real-time polymerase chain reaction (qRTPCR): Total RNA was extracted from serum using the miRNAeasy kit (Qiagen, NV, Finlow, Netherlands). The RNA samples $(5 \mu \mathrm{l})$ were diluted 20 times with ultrapure water. The purity of RNA was determined by the ratio of the ultraviolet absorbance at 260 and $280 \mathrm{~nm}$. The ratio of 1.7 to 2.1 showed that the samples had high purity and could meet the needs of further research. The cDNA template was generated by reverse transcription with a PCR amplifier. qRT-PCR was conducted by ABI 7500 quantitative PCR System (Thermo Fisher Scientific, Waltham, MA, USA) for detection of miR-21 expression. The reaction conditions were as follows: initial denaturation at $95^{\circ} \mathrm{C}$ for $10 \mathrm{~min}$ and 40 cycles of denaturation at $95^{\circ} \mathrm{C}$ for $10 \mathrm{~s}$, annealing at $60^{\circ} \mathrm{C}$ for $20 \mathrm{~s}$, and extension at $72^{\circ} \mathrm{C}$ for 34 s. $\beta$-actin was used as an internal control. The primer sequences of miR-21 and $\beta$-actin are illustrated in Table 1 . The length of amplified product of miR-21 was $350 \mathrm{bp}$, and that of $\beta$-actin was $650 \mathrm{bp}$. The target gene fragments were amplified by gel electrophoresis, and the $\mathrm{Ct}$ 
Table 1. The Primer Sequences

\begin{tabular}{cc}
\hline Target & Primer Sequences \\
\hline miR-21 & Forward: 5'-GTGCAGGGTCCGAGGT-3' \\
& Reverse: 5'-GCCGCTAGCTTATCAGACTGGT-3' \\
3 -actin & Forward: 5'-CTCGCTTCGGCAGCACA-3' \\
& Reverse: 5'-AACGCTTCACGAATTTGCGT-3'
\end{tabular}

value was obtained by scanning the ultraviolet imaging system to calculate the relative expression of miR-21 using the $2^{-\Delta \mathrm{Ct}}$ method via the formula: ${ }^{\triangle} \mathrm{Ct}=(\operatorname{target}$ gene $\mathrm{CT}-\beta$-actin $\mathrm{CT})$. $2^{-\triangle C \mathrm{t}}$ is the relative expression quantity of miR-21.

Clinical follow up: A six-month clinical follow up after PCI was completed for all patients to evaluate whether there were presences of ISR.

Statistical analysis: SPSS 21.0 statistical software (SPSS Inc., Chicago, IL, USA) was applied for statistical analysis. The measurement data were expressed by mean and standard deviation $(\chi \pm s)$. Least significant difference $t$-test was used in comparison of two groups or averages. Pearson method was used to analyze the correlation between miR-21 expression and risk factors of ISR. The clinical significance of miR-21 expression in ISR was evaluated by establishing receiver operating characteristic (ROC) curve, calculating sensitivity, specificity, and AUC. $P<.05$ was considered to indicate a statistically significant difference.

\section{RESULTS}

Clinical characteristics and IVUS parameters of coronary artery lesions for all patients: Basic demographic and clinical and biochemical characteristics of the cohort are listed in Table 2 and Figure 2. The patients between the ISR and NISR groups did not significantly differ in the main demographic parameters (age and gender) or clinical risk factors (diabetes mellitus, AMI/UA), who had a similar extent of coronary disease (multi-vessel disease [2VD/3VD]) and similar lesion characteristics (complex lesion B2/C and diameter of implanted stents). Furthermore, they had similar main iochemical parameters (creatinine, triglyceride (TG) and HDL-cholesterol). However, total cholesterol (TC) and LDL cholesterol, TNF- $\alpha$, as well as IL-6 was significantly higher for the ISR patients.

Comparison of the parameters of IVUS between groups: The parameters for EEM, PLA, and MLA revealed the statistically significant differences in the ISR group compared with those in the NISR group, respectively (Table 2).

Comparison of expression quantity of miRNA-21 between groups: The evidences of RT-PCR showed the increased expression of miR-21 in the ISR group compared with those in the NISR group (Figure 3).

Correlation analysis: In the ISR group, the expression of serum miR-2 1 had a positive correlation with PLA and had a negative correlation with EEM and with MLA. Furthermore,
Table 2. Clinical characteristics and IVUS parameters of coronary artery lesions for all patients

\begin{tabular}{lccc}
\hline Group & ISR & NISR & $P$ \\
\hline Age (years) & $55.7 \pm 12.6$ & $52.3 \pm 13.2$ & $>.05$ \\
Male/Female & $24 / 9$ & $27 / 10$ & $>.05$ \\
Diabetes mellitus (\%) & 29.75 & 28.37 & $>.05$ \\
Creat (umol/L) & $78.3 \pm 23.9$ & $75.1 \pm 22.6$ & $>.05$ \\
TC (mmol/L) & $4.73 \pm 0.56$ & $4.17 \pm 0.45$ & $<.01$ \\
TG (mmol/L) & $1.87 \pm 0.37$ & $1.79 \pm 0.36$ & $>.05$ \\
LDL-C (mmol/L) & $3.02 \pm 0.29$ & $2.37 \pm 0.23$ & $<.01$ \\
HDL-C (mmol/L) & $1.19 \pm 0.15$ & $1.15 \pm 0.13$ & $>.05$ \\
TNF- $\alpha(\mathrm{pg} / \mathrm{ml})$ & $7.3 \pm 2.6$ & $5.5 \pm 2.2$ & $<.01$ \\
IL-6 (ng/L) & $0.52 \pm 0.05$ & $0.40 \pm 0.04$ & $<.01$ \\
AMl/UA & $15 / 18$ & $17 / 20$ & $>.05$ \\
Multi-vessel disease (\%) & 53.5 & 52.7 & $>.05$ \\
B2/C lesions (\%) & 67.3 & 66.2 & $>.05$ \\
Stent diameter (mm) & $3.0 \pm 0.79$ & $3.0 \pm 0.66$ & $>.05$ \\
EEM (mm ${ }^{2}$ ) & $9.97 \pm 2.17$ & $13.66 \pm 2.28$ & $<.01$ \\
PLA (mm ${ }^{2}$ ) & $11.28 \pm 2.75$ & $6.13 \pm 1.90$ & $<.01$ \\
MLA (mm ${ }^{2}$ ) & $3.78 \pm 1.87$ & $6.07 \pm 2.07$ & $<.01$ \\
\hline
\end{tabular}

Abbreviations: LDL-C: low density lipoprotein cholesterol; HDL-C: high density lipoprotein cholesterol; TNF- $\alpha$ : Tumour necrosis factor- $\alpha$; IL-6: interleukin-6; AMI: acute myocardial infarction; UA: unstable angina pectoris; EEM: external elastic membrane; PLA: plaque area; MLA: minimal lumen area; TG: triglyceride; TC: total cholesterol

a positive correlation also has been confirmed between TNF- $\alpha$ and miR-21, as well as between IL-6 and miR-21. The scatter diagrams on correlation were shown in Figure 4.

ROC curve analysis: The effect of miR-21 on diagnosing restenosis was presented in Figure 5. The under area (AUC) of ROC curve was 0.867 (95\% confidence interval (CI: 0.752 0.9577 ). The Youden index was 0.797 and the sensitivity and specificity were $89.5 \%$ and $90.2 \%$, respectively.

\section{DISCUSSION}

In this study, we explored the possible association between miR-21 expression in blood samples from patients who successfully received PCI and the incidence of ISR six months later. Our data showed that the presence of miR-21 was associated with ISR and that TNF- $\alpha$ and IL-6 levels were significantly higher in the ISR group than those in the NISR group. The analysis for IVUS revealed that EEM and MLA significantly decreased, and PLA significantly increased for the ISR patients. More importantly, the miRNA-21 expression had a significant correlation with EEM, PLA, MLA, TNF- $\alpha$, and IL-6 1 in the ISR group, respectively. An AUC 

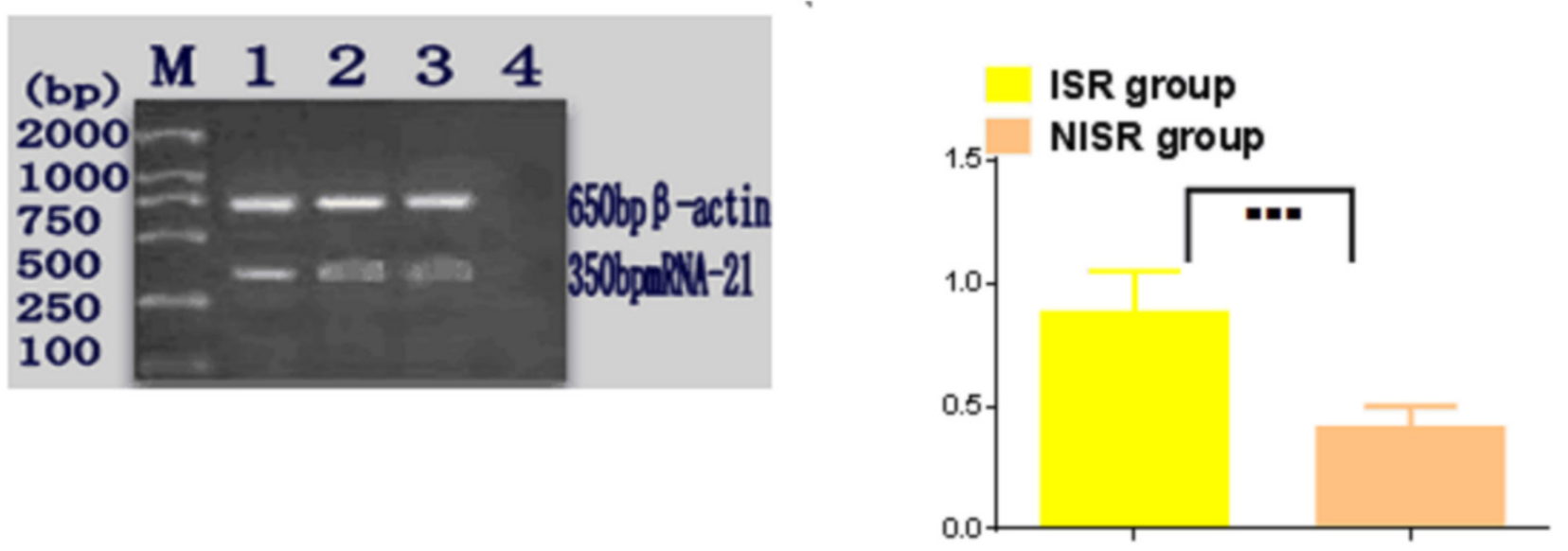

\section{miRNA-21}

Figure 3. The electrophoresis figure of the amplified target gene fragments. M, 1, 2, 3, and 4, respectively, show the expression of those in the marker (DL 2000), ISR group, NISR group, NISR group and Negative control. In ISR group and non-ISR group, the expression levels of miRNA-21 were, respectively, $(0.88 \pm 0.17,0.41 \pm 0.09)$. The expression levels of miRNA-21 significantly increased in the ISR group compared with those in the NISR group $(P<.01)$.

$\square, P<.01$

of 0.867 (95\% confidence interval (CI): 0.752-0.957) with Youden index of 0.797 , sensitivity of $89.5 \%$, and specificity of $90.2 \%$ was described.

VSMC proliferation and miRNA-21: Currently, coronary stenting intervention is used for the treatment of ischemic heart disease caused by coronary stenosis. The early diagnosis of ISR has become the focus of current research. The mechanisms of restenosis are not fully understood yet. It is widely accepted that vascular inflammation plays a central role in the pathogenesis of ISR. This condition stimulates excessive vascular smooth muscle cells (VSMCs) proliferation and migration as well as extracellular matrix remodeling, resulting in neointimal hyperplasia and eventually ISR [Donners 2003; Drachman 2005; Schillinger 2005].

Similar study also has shown that the persistence of vascular damage during PCI and bare-metal stent implantation lead to complex inflammatory and repair processes. This inflammatory response promotes the release of some leukocyte related growth factors, which leads to the further proliferation of vascular smooth muscle cells, migration to neointima, and then the formation of extracellular matrix. Excessive VSMC proliferation and extracellular matrix formation lead to neointimal hyperplasia, which represents the major pathophysiologic mechanism of ISR [Pleva 2015].

MiR-21 is a subtype of miRNA family, which has a typical gene coding region and is not affected by other coding region gene promoters and has its own promoter region, it shows over expression under various pathological conditions (such as cancer, cardiovascular disease, etc.) [Cai 2004; Fujita 2008; Lagos-Quintana 2002]. Current research suggests that miR-21 has different roles in different heart diseases because they are expressed in vascular smooth muscle, endothelial cells, cardiac myocytes, and cardiac fibroblasts [Song 2012; Sabatel 2011; Qin 2012; Liang 2012].

Increasing evidences indicated that adenovirus-mediated miR-21 sponge could significantly decrease the expression of miR-21 in cultured VSMCs and VSMC proliferation. The further analysis revealed that the phosphatase and tensin homolog (PTEN) was a potential target gene and involved in the miR-21-mediated effect on neointimal hyperplasia in vein grafts. Overexpression of miR-21 induced the proliferation of VSMCs in the aortic wall predominantly through the inhibition of PTEN [Wang 2017]. It previously has been demonstrated that the gene expression of miR-21 raised obviously after aortic balloon angioplasty in some rats [Ji 2007]. In the experiment, researchers removed miR-21 from smooth muscle cells and found that cell proliferation decreased with the increase of apoptosis. Therefore, it is thought that miRNA-21 plays an important role in promoting proliferation and anti-apoptosis in smooth muscle cells, thereby affecting the formation of neointima and participating in the pathological process of vascular stenosis.

In the present study, the expression of miR-21 increased significantly in the ISR group than those in the NISR group $(P<.01)$. These findings suggest that coronary intervention cause proliferation for the intima of the blood vessels and that ISR patients are more likely to suffer from intimal damage than those without stenosis patients. The above evidence indicates that increased miR-21 levels contribute to the effect of ISR.

Inflammation and miRNA-21: Many cytokines have been shown to increase the incidence of recurrent stenosis in the dilated segment of the coronary artery [Libby 2003; Casscells 1994; Danenberg 2003]. TNF- $\alpha$, as one of the most 


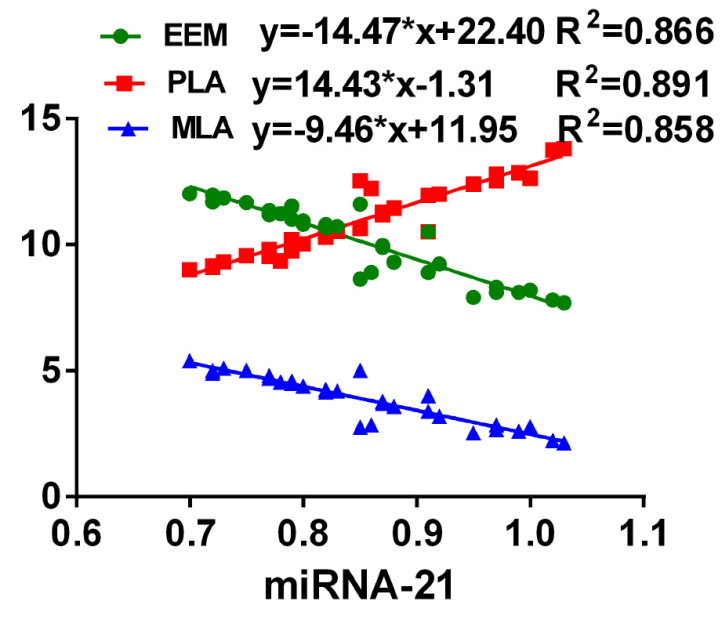

TNF- $\alpha \quad y=14.29^{*} x-5.17 R^{2}=0.952$

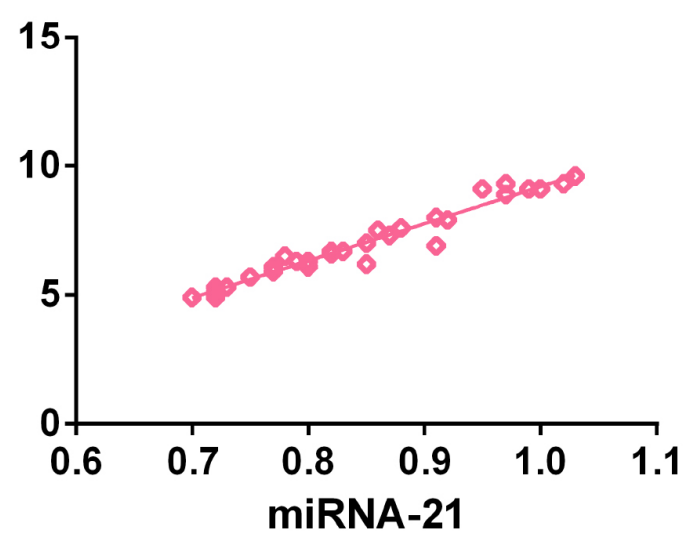

? IL-6 $y=0.27^{*} x+0.30 R^{2}=0.907$

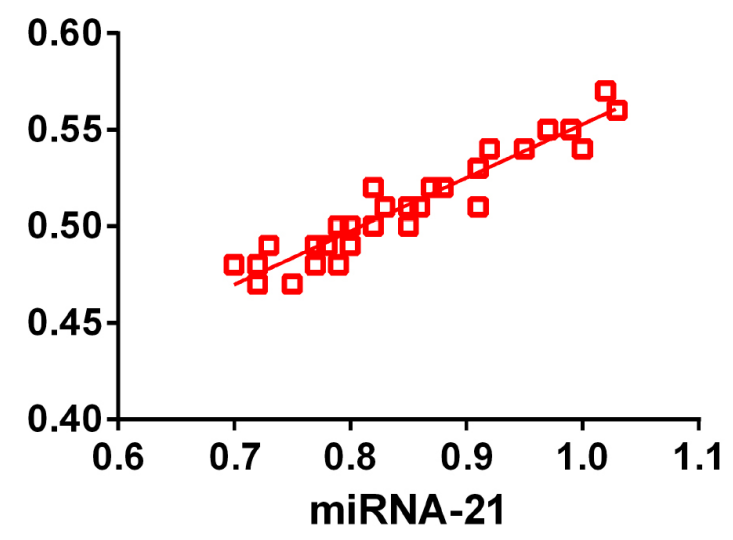

Figure 4. The scatter diagram on correlation of miRNA-21 with PLA, EEM, MLA, TNF- $\alpha$ and IL-6, respectively. $P<.01$

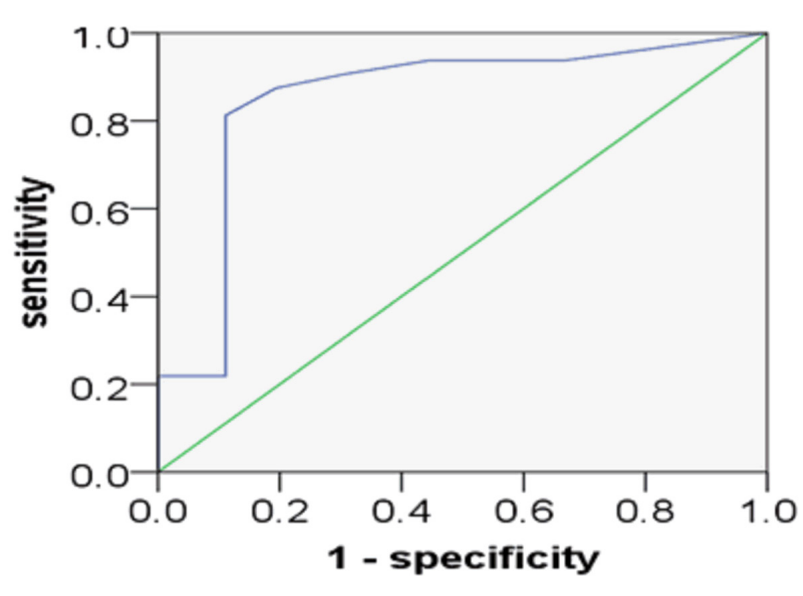

Figure 5. The ROC curves of miRNA-21

contributing inflammatory cytokines, is involved in the development of heart failure, myocardial ischemia/reperfusion injury, and coronary micro-embolization [Skyschally 2007; Schulz 2004; Chen 2014]. The elevated TNF- $\alpha$ concentrations in the myocardia following ischemia, during coronary micro-embolization or other conditions, correlate with the development of contractile dysfunction [Cao 2015]. In a recent study, TNF- $\alpha$ was chosen as a downstream target of miR-21. The study demonstrated that miR-21 regulated the expression of TNF- $\alpha$ in HeLa cells of cervical cancer cells [Xu 2017].

An increase in IL-6 level within several hours after the procedure was observed for PCI patients. However, after the first month following PCI, a decrease in IL-6 level was observed. This revealed that PCI improved myocardial ischemia and confirmed the effect of PCI. As time goes on, the decline in IL-6 level could be reversed, which also had been shown to stimulate restenosis with PCI [Hojo 2000; Szkodzinski 2009; Ikeda 2001]. Another study showed significant differences in TNF- $\alpha$, IL-6, and CRP levels between patients with troponin $\mathrm{t}$-positive and those with troponin $\mathrm{t}$-negative after selective PCI [Bonz 2003]. Shen et al. found with miR-21 upregulation, IL-6 could stimulate the anti-apoptotic IL-6/Stat3 pathway, increase cellular proliferation, and reduce apoptosis [Shen 2009].

In the present study, the levels of TNF- $\alpha$ and IL-6 increased more significantly in the ISR group. Further analysis revealed that the expression of $\mathrm{miR}-21$ had a positive correlation with the levels of TNF- $\alpha$ and IL-6. This evidence confirms that the pathogenesis of ISR might be related to inflammation, and miRNA-21 played a controlling role in the pathogenesis of ISR.

Ischemic heart disease and miRNA-21: Restenosis after PCI often is accompanied by recurrence of angina and myocardial ischemia [Alraies 2017]. Ischemia-driven target lesion revascularization (TLR) was also the same in patients randomly assigned to sirolimus-eluting stents or paclitaxel-eluting stents (13.1\% versus $15.1 \%$ ) in the study [Räber 2011]. 
In the evaluation of ischemic heart disease, intravascular ultrasound (IVUS) is a method that can directly show the stenosis of the coronary artery and accumulation of plaque, and with the least invasion [Lindsey 2016]. Conventional grayscale (B-mode) IVUS imaging helps to determine the extent and shape of plaque [Mintz 2001], guide percutaneous coronary intervention (PCI), and evaluate stent placement in subsequent imaging [Maehara 2009]. Previous study has shown that the release of vascular and myocardial miRNAs into the circulation in patients with stable CAD depend on the prevalence of significant coronary stenosis with coronary ischemia. Patients with a significant coronary stenosis showed significantly increased levels of circulating miR-21, miR-126, and miR-222 [Jansen 2017]. In a murine ischemia-reperfusion model, myocardial miR-21 was upregulated in the infarct zone and triggered fibrotic infarct remodeling by phosphatase and tensin homolog [Roy 2009]. Ischemia-induced upregulation of miR-21 could present a possible underlying mechanism in patients with significant coronary stenosis and cardiac ischemia [Huang 2016].

Here, we presented evidence of IVUS for PCI patients, in which these parameters of IVUS, including EEM, MLA and PLA, presented significant differences for ISR patients. In our findings, the expression of miR-21 had significant correlation with EEM, MLA, and PLA. These findings suggested that the obvious restenosis of the coronary artery after PCI regulated the expression of circulating miRNA-21, which may be related to myocardial ischemia.

According to our results of ROC curve, AUC of serum miR-21 expression was 0.867 , associated with $89.5 \%$ sensitivity and $90.2 \%$ specificity, suggesting that miRNA-21 may be an effective marker to distinguish ISR after PCI.

The above findings indicated that miR-21 was closely related to the development of ISR after PCI and may be involved in the pathogenesis of restenosis.

\section{CONCLUSION}

The patients between the ISR and NISR groups did not significantly differ in the main demographic parameters (age and gender) or clinical risk factors (diabetes mellitus, AMI/UA), who had a similar extent of coronary disease (multi-vessel disease [2VD/3VD]) and similar lesion characteristics (complex lesion B2/C and diameter of implanted stents). Furthermore, they had similar main iochemical parameters (creatinine, TG and HDL-C). However, total TC and LDL cholesterol was significantly higher for the ISR patients.

Our results showed that the expression of miR-21 were significantly associated with ISR in the patients after PCI. The detection of the expression of serum miR-21 for forecasting of ISR is of great importance, creating a new way in the diagnosis and treatment of ISR.

Limitations: This study could be limited by a single miRNA research and relatively small sample size.
ACKNOWLEDGEMENTS

The authors thank the staff of Department of Laboratory and Department of Echocardiography from the First People's Hospital of Xian Yang City and the Eighth Hospital of Xi An City for the excellent support.

\section{REFERENCES}

Alraies MC, Darmoch F, Tummala R, Waksman R. 2017. Diagnosis and management challenges of in-stent restenosis in coronary arteries. World J Cardiol. 8:640-651.

Bentwich I, Avniel A, Karov Y, et al. 2005. Identification of hundreds of conserved and nonconserved human microRNAs, Nat Genet. $37,766-770$.

Bonz AW, Lengenfelder B, Jacobs M, Strotmann J, Held S, Ertl G, Voelker W. 2003. Cytokine response after percutaneous coronary intervention in stable angina: effect of selective glycoprotein IIb/IIIa receptor antagonism. Am Heart J. 145:693-699.

Cai X, Hagedorn CH, Cullen BR. 2004. Human microRNAs are processed from capped, polyadenylated transcripts that can also function as mRNAs, RNA. 10, 1957- 1966.

Cao YY, Chen ZW, Gao YH, Wang XX, Ma JY, Chang SF, Qian JY, Ge JB. 2015. Exenatide Reduces Tumor Necrosis Factor- $\alpha$-induced Apoptosis in Cardiomyocytes by Alleviating Mitochondrial Dysfunction. Chin Med J (Engl). 23: 3211-3218.

Casscells W, Roberts R, Towbin JA. 1994. Mechanisms of restenosis. Texas Heart Inst J. 21:68-77.

Chen ZW, Qian JY, Ma JY, Chang SF, Yun H, Jin H, et al. 2014. TNFa-induced cardiomyocyte apoptosis contributes to cardiac dysfunction after coronary microembolization in mini-pigs. J Cell Mol Med. 18: 1953-1963.

Danenberg HD, Szalai AJ, Swaminathan RV, Peng L, Chen Z, Seifert P, Fay WP, Simon DI, Edelman ER. 2003. Increased thrombosis after arterial injury in human C-reactive protein-transgenic mice. Circulation. 108: $512-515$.

Donners MM, Daemen MJ, Cleutjens KB, Heeneman S. 2003. Inflammation and restenosis: implications for therapy. Ann Med. 35:523-531.

Drachman DE, Simon DI. 2005. Inflammation as a mechanism and therapeutic target for in-stent restenosis. Curr Atheroscler Rep. 7:44-49.

Fichtlscherer S, De Rosa S, Fox H, Schwietz T, Fischer A, Liebetrau C, Weber M, Hamm CW, Roxe T, Muller-Ardogan M, Bonauer A, Zeiher AM, Dimmeler S. 2010. Circulating microRNAs in patients with coronary artery disease. Circ Res. 107:677-684.

Fichtlscherer S, Zeiher AM, Dimmeler S. 2011. Circulating microRNAs: biomarkers or mediators of cardiovascular diseases? Arterioscler Thromb Vasc Biol. 31:2383-2390.

Fujita S, Ito T, Mizutani T, et a1. 2008. MiR-21 Gene expression triggered by AP-1 is sustained through a double-negative feedback mechanism. JMol Biol. 378, 492-504.

Hojo Y, Ikeda U, Katsuki T, Mizuno O, Fukazawa H, Kurosaki K, Fujikawa H, Shimada K. 2000. Interleukin 6 expression in coronary circulation after coronary angioplasty as risk factor for restenosis. Heart. 84:83-87.

Huang W, Tian SS, Hang PZ, Sun C, Guo J, Du ZM. 2016. Combination of microRNA-21 and microRNA-146a Attenuates Cardiac Dysfunction 
and Apoptosis During Acute Myocardial Infarction in Mice. Mol Ther Nucleic Acids. 3:e296.

Ikeda U, Ito T, Shimada K. 2001. Interleukin-6 and acute coronary syndrome. Clinic Cardiol. 24:701-704.

Jansen F, Schäfer L, Wang H, Schmitz T, Flender A, Schueler R, et al. 2017. Kinetics of Circulating MicroRNAs in Response to Cardiac Stress in Patients With Coronary Artery Disease. J Am Heart Assoc. 8:e005270.

Ji R, Cheng Y, Yue J, et a1. 2007. MicroRNA expression signature and antisense-mediated epletion reveal an essential role of MicroRNA in vascular neointimal lesion formation. Circ Res. 100, 1579-1588.

Kim MS, Dean LS. 2011. In-Stent Restenosis. Cardiovasc Ther. 29:190-8.

Lagos-Quintana M, Rauhut R, Yalcin A, et a1. 2002. Identification of tissue specific micmRNAs from mouse, Curr Biol 12:735-739.

Li-Sha G, Peng C, Yue-Chun L. 2015. Recurrent acute coronary syndrome and restenosis after percutaneous coronary intervention in a patient with idiopathic thrombocytopenic purpura: a case report and literature review. BMC Cardiovasc Disord. 15:101.

Liang H, Zhang C, Ban T, et a1. 2012. A novel reciprocal loop between microRNA-2 1 and TGFbetaRIII is involved in cardiac fibrosis. Int JBiochem Cell Bio. 144:2152- 2160.

Libby P, Simon DI, Rogers C. 2003. Inflammation and artery injury. In: Topol E, editor. Textbook of interventional cardiology. Philadelphia: Saunders. pp. 381-389.

Lindsey BD, Martin KH, Jiang X, Dayton PA. 2016. Adaptive windowing in contrast-enhanced intravascular ultrasound imaging. Ultrasonics. 70:123-135.

Ma N, Bai J, Zhang W, Luo H, Zhang X, Liu D, Qiao C. 2016. Trimetazidine protects against cardiac ischemia/reperfusion injury via effects on cardiacmiRNA-21 expression, Akt and the Bcl 2/Bax pathway. Mol Med Rep. 5:4216-4222.

Maehara A, Mintz GS, Weissman NJ. 2009. Advances in intravascular imaging. Circ Cardiovasc Interv. 2:482-490.

Mintz GS, Nissen SE, Anderson WD, Bailey SR, Erbel R, Fitzgerald PJ, et al. 2001. American College of Cardiology Clinical Expert Consensus Document on Standards for Acquisition, Measurement and Reporting of Intravascular Ultrasound Studies (IVUS). A report of the American College of Cardiology Task Force on Clinical Expert Consensus Documents. J Am Coll Cardiol. 37:1478-1492.

Mitchell PS, Parkin RK, Kroh EM, et a. 2008. Circulating microRNAs as stable blood-based markers for cancer detection, Proc Natl Acad Sci USA. 105:10513- 10518 .

Pleva L, Kovarova P, Faldynova L, Plevova P, Hilscherova S, Zapletalova J, Kusnierova P, Kukla P. 2015. The rs1803274 polymorphism of the BCHE gene is associated with an increased risk of coronary in-stent restenosis. BMC Cardiovasc Disord. 15:135.

Qiang S, checked by Lang L. 2013. The research progress of relationship between microRNA-21 and ischemic heart disease. Chin Circ. 28,390-392.

Qin Y, Yu Y, Dong H, et a1. 2012. MicroRNA 21 inhibits left ventricular remodeling in the early phase of rat model with ischemia-reperfusion injury by suppressing cell apoptosis, Int J Med Sci. 9:413-423.
Räber L, Wohlwend L, Wigger M, Togni M, Wandel S, Wenaweser P, Cook S, Moschovitis A, Vogel R, Kalesan B, Seiler C, Eberli F, Lüscher TF, Meier B, Jüni P, Windecker S. 2011. Fiveyear clinical and angiographic outcomes of a randomized comparison of sirolimuseluting and paclitaxeleluting stents: results of the SirolimusEluting Versus PaclitaxelEluting Stents for Coronary Revascularization LATE trial. Circulation $123: 28192828$.

Ren Y, Chen KJ, Ruan XM. 2008. [Systematic review of randomized controlled trials on preventing and treating restenosis after percutaneous coronary intervention with Chinese medicine]. [Article in Chinese]. Zhongguo Zhong Xi Yi Jie He Za Zhi. 28: 597-601.

Roy S, Khanna S, Hussain S-RA, Biswas S, Azad A, Rink C, Gnyawali S, Shilo S, Nuovo GJ, Sen CK. 2009. MicroRNA expression in response to murine myocardial infarction: miR-21 regulates fibroblast metalloprotease-2 via phosphatase and tensin homologue. Cardiovasc Res. 82:21-29.

Ryan TJ, Faxon DP, Gunnar RM, Kennedy JW, King III SB, Loop FD, et al. 1988. Guidelines for percutaneous transluminal coronary angioplasty: A report of the American College of Cardiology/American Heart Association Task Force on the Assessment of Diagnostic and Therapeutic Cardiovascular Procedures. Circulation. 2:486-502.

Sabatel C, Malvaux L, Bovy N. et a1. 2011. MicroRNA-21 exhibits antiangiogenic function by targeting RhoB expression in endothelial cells. Plos One. 6:e16979.

Schillinger M, Minar E. 2005. Restenosis after percutaneous angioplasty: the role of vascular inflammation. Vasc Health Risk Manag. 1:73-78.

Schulz R, Aker S, Belosjorow S, Heusch G. 2004. TNFalpha in ischemia/ reperfusion injury and heart failure. Basic Res Cardiol. 99: 8- 11.

Shen XH, Han YJ, Zhang DX, Cui XS, Kim NH. 2009. A link between the Interleukin-6/Stat3 anti-apoptotic pathway and microRNA-21 in preimplantation mouse embryos. Mol Reprod Dev. 76: 854-862.

Skyschally A, Gres P, Hoffmann S, Haude M, Erbel R, Schulz R, et al. 2007. Bidirectional role of tumor necrosis factor-alpha in coronary microembolization: Progressive contractile dysfunction versus delayed protection against infarction. Circ Res. 100:140-146.

Small EM, Olson EN. 2011. Pervasive roles of microRNAs in cardiovascular biology. Nature. 469:336-342.

Song J, Hu B, Qu H, et a1. 2012. Mechanical stretch modulates microRNA 21 expression, participating in proliferation and apeptosis in cultured human aortic smooth muscle cells, Plos One. 7:e47657.

Szkodzinski J, Blazelonis A, Wilczek K, Hudzik B, Romanowski W, Gasior M, Wojnar R, Lekston A, Polonski L, Zubelewicz-Szkodzinska B. 2009. The role of interleukin-6 and transforming growth factor- 1 in predicting restenosis within stented infarct-related artery. Int J Immunopathol Pharmacol. 22: 493-500.

Wang XW, Zhang C, Lee KC, He XJ, Lu ZQ, Huang C. 2017. Adenovirus-Mediated Gene Transfer of microRNA-21 Sponge Inhibits Neointimal Hyperplasia in Rat Vein GraftsInt J Biol Sci. 10, 1309-1319.

Xu L, Xu Q, Li X, Zhang X. 2017. MicroRNA-21 regulates the proliferation and apoptosis of cervical cancer cells via tumor necrosis factor- $\alpha$. Mol Med Rep. 4:4659- 4663.

Yang SJ, Zhang Y, Liu Y. 2015. Research on expression of miRNA-21 in the peripheral blood of coronary heart disease and its clinical significance. Zhong Guo Ying Yong Sheng Li Xue Za Zhi. 2:127- 31. 